\title{
A new shoulder orthosis to dynamically support glenohumeral subluxation
}

\author{
Claudia J. W. Haarman", Edsko E. G. Hekman, Member, IEEE, Martijn F. H. Haalboom, Herman van \\ der Kooij, Member, IEEE, and Johan S. Rietman
}

\begin{abstract}
Objective: In this paper we presented a novel shoulder subluxation support that aims to reduce the stress on the passive structures around the shoulder of patients with glenohumeral subluxation and glenohumeral-related shoulder pain. The device applies a force to the upper arm without impeding the functional range of motion of the arm. Our design contains a mechanism that statically balances the arm with two elastic bands. Methods: A technical evaluation study was conducted to assess the performance of the orthosis. Additionally, two patients evaluated the orthosis. Results: The results of the technical validation confirms the working of the balancing mechanism. The pilot study demonstrated that the shoulder support increased the feeling of stability of the shoulder joint and, to a lesser extent, decreased shoulder pain. Furthermore, both patients reported that the orthosis did not impede their range of motion. Conclusion: In this research we developed a shoulder orthosis based on two statically balanced springs that support the shoulder of patients with glenohumeral subluxation that have residual shoulder muscle force. Compared to existing shoulder supports, our design does not impede the range of motion of the arm, and continues to provide a stabilizing force to the shoulder, even if the arm is moved away from the neutral position. Test with two participants showed promising results. Significance: The device presented in this work could have a significant impact on the shoulder function which may improve rehabilitation outcome and improve the quality of life of patients suffering from glenohumeral subluxation and shoulder pain.
\end{abstract}

Index Terms-Orthotics, assistive device, upper extremity exoskeleton, passive mechanism, shoulder pain, shoulder subluxation.

\section{INTRODUCTION}

$\mathrm{T}$ HE shoulder complex contributes to the mobility necessary to perform reaching tasks. The glenohumeral joint $(\mathrm{GHJ})$ is one of the four joints that together form the shoulder complex. The GHJ is a ball and socket joint that allows for a large range of motion of the upper arm. As a consequence, this joint is relatively unstable [1]. For its stability the GHJ heavily relies on the posterior deltoid and the supraspinatus,

Copyright (c) 2017 IEEE. Personal use of this material is permitted. However, permission to use this material for any other purposes must be obtained from the IEEE by sending an email to pubs-permissions@ieee.org.

This project has received funding from the European Unions Horizon 2020 research and innovation programme under grant agreement No. 688857 (SoftPro).

*C.J.W. Haarman is with the Department of Biomechanical Engineering, University of Twente, Enschede, the Netherlands, (correspondence e-mail: together with the other rotator cuff muscles (infraspinatus, teres minor, and subscapularis) and capsule and ligaments [2]. Without normal tone of (one or more of) these muscles, the contribution of the relatively weak inferior and anterior capsule and ligaments to the stabilization of the shoulder is increased. These structures are easily overstretched due to the weight of the arm [3]. An inferior displacement of the humeral head from glenoid fossa may occur, also referred to as glenohumeral subluxation (GHS). The continuous stretching of the capsule and ligaments can be painful [4]. GHS often leads to a reduction of the painless range of motion of the shoulder, thus inhibiting the functional recovery of the upper limb and compromising the arm function during daily activities [5].

Shoulder supports, also known as braces or orthoses, are frequently prescribed to patients with GHS [6]-[8]. Clinical reasons to prescribe a shoulder brace include, among others, a reduction of stress in the passive structures from gravitational pull during standing or walking, maintenance of a proper glenohumeral alignment and shoulder pain reduction [9]. Several orthoses are commercially available, including: Omo Neurexa (Ottobock, Germany) [10], Neuro-Lux (Sporlastic GmbH, Germany) [11] and GivMohr (GivMohr Corporation, US) [12]. The aim of these ortheses is to reduce stress from the gravitational pull by applying an external force between the humerus and scapula that pulls the arm upwards. Stress reduction is closely related to proper GHJ alignment. A recent review by Nadler et al. concluded that, on average, shoulder orthoses are able to reduce the vertical subluxation with at least 5 to $10 \mathrm{~mm}$ in neutral position [6]. This instantaneous mechanical effect of shoulder braces is often assessed by measuring the humeral head position relative to the scapula from an X-ray with the arm in neutral position after initial fitting.

Several studies assessed the effect of a shoulder brace on shoulder pain reduction [10],[11],[13]. Across these studies, only a modest improvement in pain measures is reported by $57 \%$ of the patients who used the orthosis for a few weeks.

c.j.w.haarman@utwente.nl) and with Hankamp Rehab, Enschede, the Netherlands.

E.E.G. Hekman, M.F.H. Haalboom, and H. van der Kooij are with the Department of Biomechanical Engineering, University of Twente, Enschede, the Netherlands.

J.S. Rietman is with the Department of Biomechanical Engineering, University of Twente, Enschede, the Netherlands, with the Roessingh, Center for Rehabilitation, Enschede, the Netherlands and with the Roessingh, Research and Development, Enschede, the Netherlands. 
Besides the low evidence for efficacy of shoulder supports [8],[12],[14], other commonly observed disadvantages include a reduction of the functional range of motion of the arm, due to the use of non-elastic straps in many braces. These straps create an upward force on the upper arm in resting position, but resist arm movement and thus limit the remaining range of motion. Van Bladel et al. stress the importance of actively engaging the arm in muscle activity to preserve glenohumeral alignment [13].

In this paper we present the concept of a new shoulder subluxation orthosis that reduces the stress on the soft tissue around the shoulder complex of patients with GHS and GHSrelated shoulder pain without limiting the retaining range of motion of the arm, such that the functional recovery of patients with remaining arm function is not inhibited and shoulder pain is reduced. The orthosis is suitable for, but not limited to, patients suffering from rotator cuff injury, stroke or brachial plexus injury.

We propose a design that consists of a trunk part and an upper arm cuff, connected only by two elastic bands (there is no hinge). It applies a force to the upper arm, directed towards the center of rotation $(\mathrm{CoR})$ of the glenoid across the entire functional range of motion. Our proposed mechanism uses static balancing of two elastic bands to achieve this (Fig. 1).

\section{DESIGN CRITERIA}

The aim of the shoulder subluxation support is to reduce shoulder pain by reducing the stress on the passive structures surrounding the shoulder complex without impeding the functional range of motion of the arm.

In order to take over the function of the passive structures surrounding the shoulder joint, the shoulder orthosis should be able to apply an upward force with a magnitude of at most one times the mass of the arm. This equals a supporting force that

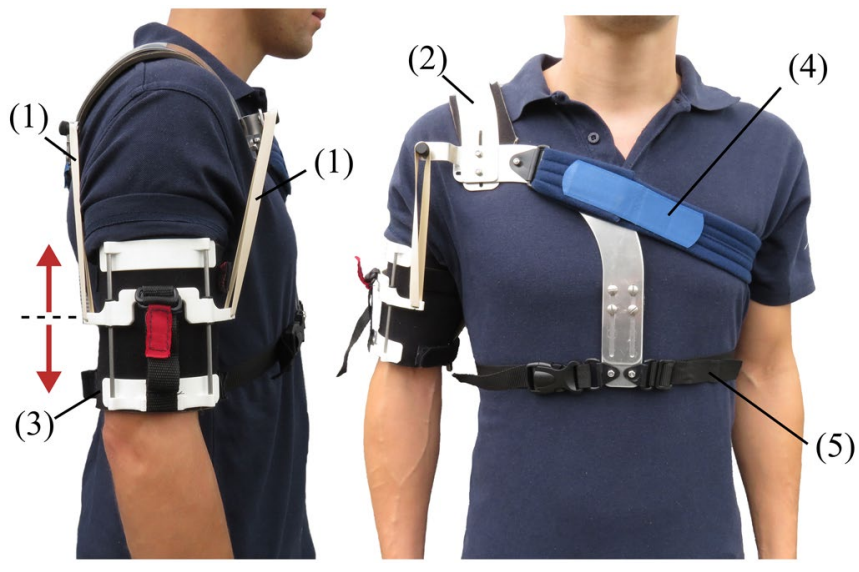

Fig. 1. Sagittal and frontal view of the prototype of the shoulder orthosis, consisting of five components: (1) Anterior and posterior elastic bands. (2) Shoulder bracket that provides a stable base for the two proximal elastic band attachment points. (3) An upper arm cuff with tensioning mechanism provides a stable base for the two distal elastic band attachment points. The amount of force applied to the upper arm is controlled by adjusting the length and/or number of the elastic bands. Sliding of the arm cuff with respect to the skin is prevented by gel liner material on the inside of the arm cuff. Users will be instructed how to properly put on the arm cuff. The red arrows indicate the allowed movement of the white 3D printed slider. (4) Strap to the contralateral shoulder, and (5) strap around the waist that keep the shoulder bracket in place.
TABLE I

MAIN REQUIREMENTS OF THE SHOULDER SUBLUXATION BRACE

\begin{tabular}{l}
\hline \hline Requirement \\
\hline - Allowed range of motion by mechanism: \\
$\circ \quad$ Up to $90^{\circ}$ shoulder elevation (or higher) \\
$\circ \quad$ Up to $20^{\circ}$ shoulder extension (or higher) \\
- Force applied to the upper arm \\
$\circ \quad$ In the direction of the glenoid: \\
$\circ \quad$ Up to $100 \%$ of arm weight \\
$\circ \quad$ During entire allowed range of motion \\
- Prevent added resistance by brace during movement \\
- Prevent synergistic flexion patterns and soft tissue contractures \\
- Minimize visibility
\end{tabular}

completely reduces the gravitational pull on the arm and is considered as a worst-case scenario. Depending on the condition of the patient (e.g. residual muscle force) and individual preferences, less supporting force may be required. Therefore, the user must be able to easily adjust the magnitude of this upward force.

To not impede the functional range of motion of the arm, the supportive force should be directed towards the center of rotation of the glenoid. Several studies investigated arm movements (frequency and elevation levels) of healthy individuals during daily activities [15]-[17]. On average, about $90 \%$ of the arm movements during daily activities involve flexion angles less than $90^{\circ}$. Also, the time spent below a flexion angle of $90^{\circ}$ was approximately $96 \%$ of the time [18],[19]. The brace should therefore allow for an elevation angle of at least $90^{\circ}$.

The brace should not obstruct normal arm swing, since disturbed arm swing increases the metabolic cost of walking [20],[21]. Maximum shoulder extension angles of approximately $20^{\circ}$ are seen during normal walking [20]. The device should therefore allow for a shoulder extension angle of at least $20^{\circ}$.

The achieved pain reduction with existing braces is modest. $57 \%$ of the patients across three studies show an improvement of shoulder pain [6]. Most of the commercially available braces feature two non-elastic straps on the anterior and posterior part of the arm. From a mechanical viewpoint it can be seen that these straps resist arm motion in case they have been fitted tight with the arm in a neutral position. Fitting the straps loosely in neutral arm position allows arm motion but does not provide sufficient upward force. In the latter case, the reduction of the stress on the passive structures is low, especially in this neutral position. In order to further reduce the stress on the structures around the shoulder complex, a restoring force in the direction of the glenoid should be present throughout the entire range of motion.

GHS is associated with a decreased muscle tone of the rotator cuff [22]. The residual strength of unaffected muscles should be optimally deployed. Additional effort by the patients to overcome any added resistance during movements with the shoulder brace is undesirable and should be prevented as much as possible.

The orthosis should not restrict elbow movement, to not impede functional use of the arm, and to prevent synergistic flexion patterns and soft tissue contractures of the elbow [7],[9]. 

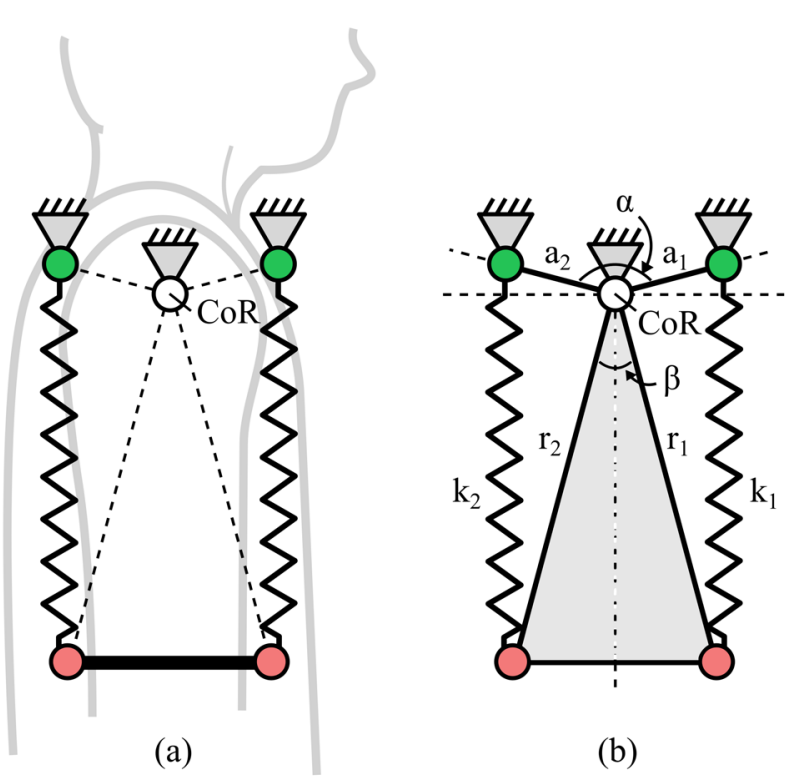

(b)

Fig. 2 (a) Principle of spring to spring balancing using a rotated spring balancer. (b) The spring balancer mechanism applied to the shoulder. Two zero-free-length springs are placed between attachment points (green and red) located on the trunk and upper arm to create a force towards the center of rotation.

Considerations when designing an orthosis include, among others, the optimization of cosmesis [23]. In order to minimize the visibility, the brace should preferably be worn under clothing. This requires a slim design that closely follow the contours of the patient's body.

In Table I a summary of the main requirements is listed.

\section{Conceptual Design}

An upward (restoring and stabilizing) force on the upper arm that will not impede the functional range of motion of the arm can be generated using two springs attached between the upper arm and the shoulder. Fig. 2(a) shows a schematic overview of this spring balancing mechanism. The proximal spring attachment points (green) are fixed to the trunk and the distal spring attachment points (red) are fixed to the upper arm.
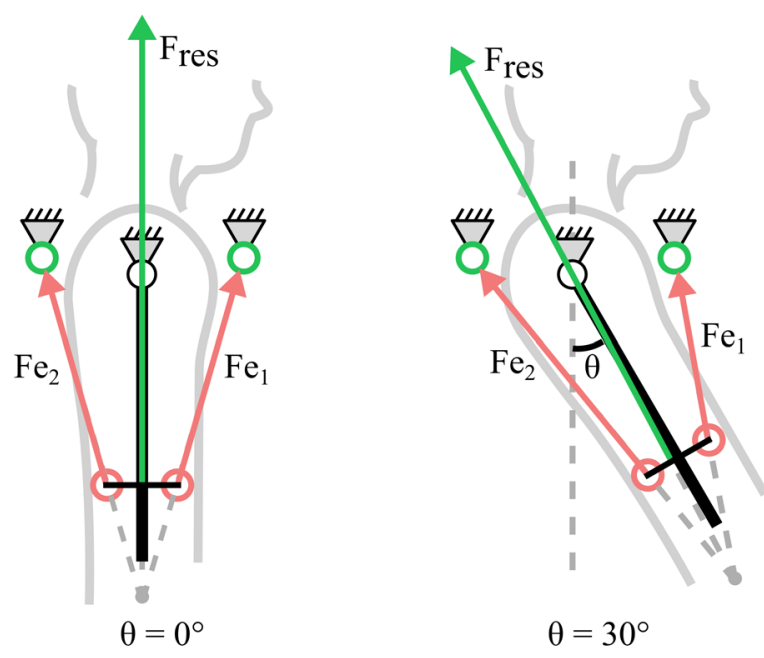

\section{A. Static Balance}

Mechanically, the shoulder-arm-spring system resembles a spring-loaded lever, where the glenohumeral joint forms the rotation point. If the springs are balanced, the resulting force is directed towards the center of rotation of the glenohumeral joint, and no joint moment is introduced. Perfect balance of this rotated spring balancer is achieved when the following conditions are met [24]:

$$
\begin{aligned}
& \alpha+\beta=\pi \\
& k_{1} a_{1} r_{1}=k_{2} a_{2} r_{2}
\end{aligned}
$$

Where $k_{1}$ and $k_{2}$ are the spring stiffnesses, $a_{1}$ and $a_{2}$ are the distances from the center of rotation to the proximal spring attachment points, and $r_{1}$ and $r_{2}$ the distances from the rotation center to the distal spring attachment points. $\alpha$ and $\beta$ are the angles between $a_{1}$ and $a_{2}$, resp. $r_{1}$ and $r_{2}$. In Fig. 2(b) the parameters are shown.

Additionally, the spring force $F$ must be proportional to its length $x$ :

$$
F=k x
$$

Springs that exhibit this behavior are called "ideal springs" or "zero-free-length springs".

\section{B. Direction Resultant Force}

The resulting force $F_{R E S}$ is in any configuration directed towards the center of rotation. In Fig. 3 three examples (upper arm flexion angles of $0^{\circ}, 30^{\circ}$ and $60^{\circ}$ ) are shown to illustrate this. Since the resultant force (green arrow) crosses the center of rotation, the resulting moment that the elastic bands apply to the GHJ is equal to zero. Arm movements in the sagittal plane are therefore not impeded by the brace. Note: since the system is always balanced under the stated conditions, regardless of position, there is no requirement for symmetry of the attachment points in vertical arm position. The direction of the resultant force cannot be changed by the user. The elastic band

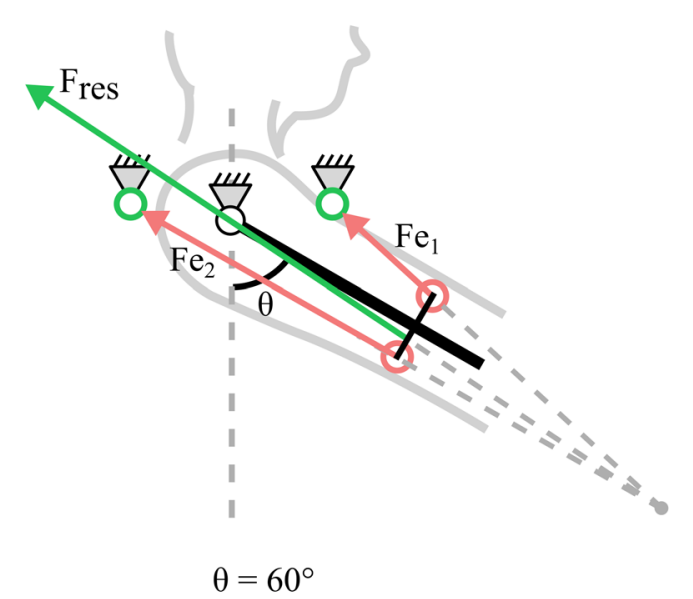

Fig. 3 The force vectors (red arrows $\mathrm{F}_{\mathrm{e} 1}$ and $\mathrm{F}_{\mathrm{e} 2}$ ) created by the elastic bands are shown for different upper arm flexion angles $\left(\theta=0^{\circ}, 30^{\circ}\right.$ and $\left.60^{\circ}\right)$. It can be seen that in each situation, the resultant force (green arrow $\mathrm{F}_{\mathrm{res}}$ ) travels through the center of rotation of the glenohumeral joint. 
TABLE II

CHARACTERISTICS OF ELASTIC BANDS 1 AND 2 USED IN THIS STUDY

\begin{tabular}{lll}
\hline \hline & \multicolumn{1}{c}{ Elastic band 1 } & \multicolumn{1}{c}{ Elastic band 2 } \\
\hline Material & Natural rubber (latex) & $\begin{array}{l}\text { Synthetic polyisopropene } \\
\text { (non-latex) }\end{array}$ \\
Free length & $88.9 \times 10^{-3} \mathrm{~m}$ & $88.9 \times 10^{-3} \mathrm{~m}$ \\
Width & $12.7 \times 10^{-3} \mathrm{~m}$ & $6.4 \times 10^{-3} \mathrm{~m}$ \\
Thickness & $8 \times 10^{-4} \mathrm{~m}$ & $8 \times 10^{-4} \mathrm{~m}$
\end{tabular}

TABLE III

CONSTRAINTS OF ELASTIC BAND ATTACHMENT POINT LOCATIONS

\begin{tabular}{lll}
\hline \hline Constraint & Symbol & Value \\
\hline $\begin{array}{l}\text { Maximum distance from skin } \\
\text { surface }\end{array}$ & Rmax & $30 \times 10^{-3} \mathrm{~m}$ \\
$\begin{array}{l}\text { Minimum shoulder flexion angle } \\
\text { Minimum shoulder extension }\end{array}$ & $\theta \mathrm{f}$ & $90^{\circ}$ \\
$\begin{array}{l}\text { angle } \\
\begin{array}{l}\text { Distrance from CoR to distal } \\
\text { spring attachment point }\end{array}\end{array}$ & $\mathrm{L}$ & $20^{\circ}$ \\
Elastic bands & & $0.2 \mathrm{~m}$ \\
& & $\begin{array}{l}\text { Should have minimal } \\
\text { contact with the skin }\end{array}$
\end{tabular}

attachment points will be aligned only once during the initial fitting process by the orthotist.

\section{Spring Tensioning Mechanism}

The magnitude of this supporting force can be manually adjusted by the user with the elastic band tensioning mechanism mounted on the arm cuff, see also Fig. 1. Pulling on a strap with their unaffected hand will allow users to move a carriage containing the distal spring attachment points over two linear guides. The distal spring attachment points can be moved

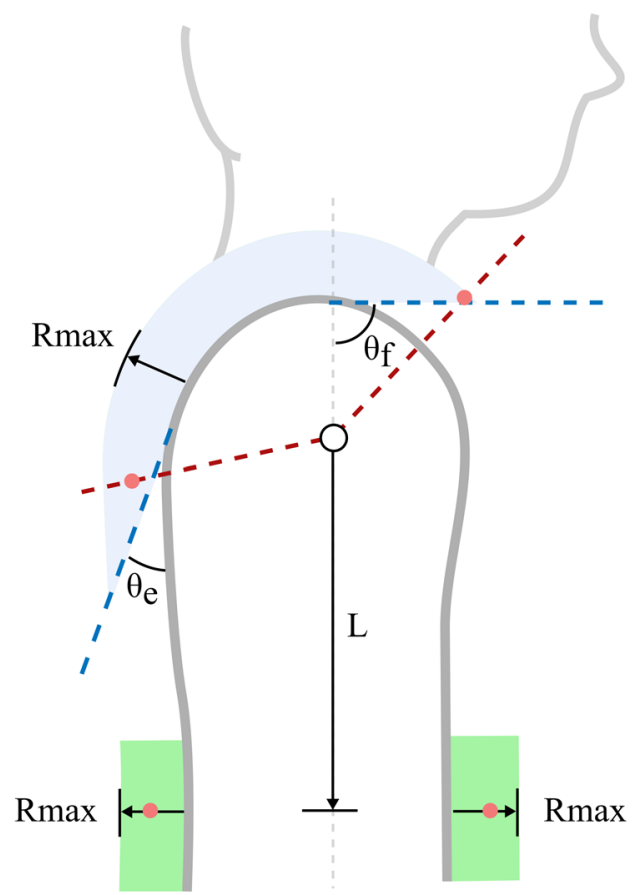

Fig. 4 Sagittal view of the upper arm. The blue shaded area shows the valid area for the proximal spring attachment locations and is bounded by the allowable distance from the skin surface (Rmax) and shoulder flexion ( $\theta f)$ and extension $(\theta \mathrm{e})$ angles. The green shaded area shows the valid area for the distal spring attachment locations and is bounded by Rmax. The red dots show a possible solution of the attachment point locations for the 50th percentile male. For 90 percent of the adult population, the proximal spring attachment points $\mathrm{P}_{1}$ and $\mathrm{P}_{2}$ are always located on the red dashed line. approximately $5 \mathrm{~cm}$ up and down. This will alter the supportive (upward) force of the elastic bands.

\section{Zero-free-length Springs}

Zero-free-length springs, necessary to achieve balance (see also (3)), have a force proportional to their length. Elastic bands made from natural rubber or synthetic polyisoprene (non-latex rubber) are a practical implementation of zero-free-length springs when used in their linear range, which is typically between $150 \%$ and $350 \%$ of their initial length [25]. The characteristics of the two types of elastic bands used in this study are summarized in Table II. These commercially available bands are composed of different materials and have a different stiffness. The selected stiffness of the elastic bands can, together with their length adjustment, provide a wide range of supportive forces. For example, elastic band I can support between 31 and $50 \%$ of the arm weight of a 50th percentile male, whereas elastic band II can support between 44 and $71 \%$ of the arm weight. The elastic bands are chosen such that their ranges overlap. If required, a combination of multiple springs can increase the supportive force range even further. In the section about the elastic bands their lifetime, stiffness and linearity over time are further characterized.

\section{METHODS}

In this section, we propose methods to validate the technical performance of the orthosis and evaluate the orthosis with patients.

\section{A. Attachment Point Locations}

The attachment point locations of the elastic bands should respect the conditions for static balance, remain close to the body, and not impede the functional range of motion of the upper arm (between $-20^{\circ}$ and $90^{\circ}$ arm flexion). The latter means that the arm should not touch the proximal attachment points within the allowed range of motion. In order to wear the brace

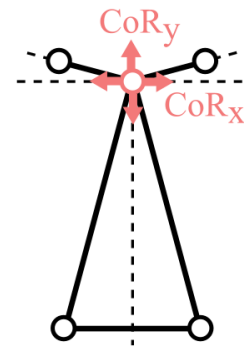

(a)

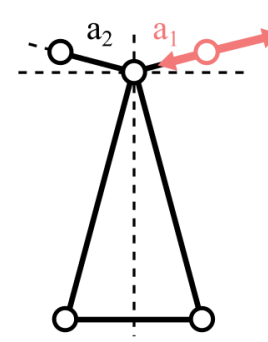

(b)

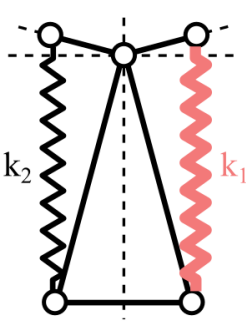

(c)

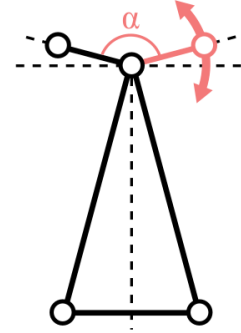

(d)

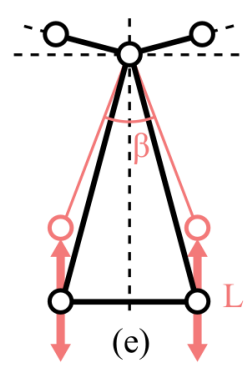

Fig. 5 Parameters that are changed to investigate static balance of the system. a) Mechanism center of rotation shifted in $x$ - and $y$-direction. b) Changing distance $a_{1}$ with respect to $a_{2}$. c) Changing stiffness $k_{1}$ with respect to $k_{2}$. d) Changing angle $\alpha$ e) Changing angle $\beta$ by varying length $L$. 
under clothing, the brace should have maximum height of $3 \mathrm{~cm}$ ( $\mathrm{R}_{\mathrm{MAX}}$ ) added to the skin surface. The constraints are summarized in Table III and visually depicted in Fig. 4. Note that the constraints resulting from the conditions for static balance are not included in this table.

A custom Matlab script was used (MathWorks, USA) to search for attachment point locations that satisfy the conditions for balance and constraints. Preferably, solutions are chosen where the elastic band does not touch the arm, to reduce friction between the elastic bands and the skin. Feasible combinations of model parameters that respect all constraints are investigated for similarity to obtain a shoulder brace design that is easily customizable to 90 percent $\left(5^{\text {th }}\right.$ to $95^{\text {th }}$ percentile) of the adult population. Anthropometric models of this population were constructed using normative data from multiple sources [26],[27].

\section{B. Conditions for Balance}

The shoulder brace should be properly aligned to minimize the added resistance by the brace to arm movements. A sensitivity analysis is performed to investigate the sources of misalignment that potentially degrade the balance of the system. For this analysis a fixed reference geometry $\left(50^{\text {th }}\right.$ percentile male) is used and only mechanism parameters are changed.

Static balance is compromised if (1) or (2) are violated, or if the elastic bands are used outside their zero-free-length range such that (3) is no longer valid. In the following paragraphs we investigated the influence of changing these parameters on the conditions for balance. In Fig. 5 a graphical overview of all possible types of misalignment is presented.

1) Potential Energy: Equation (2) relies on the correct positioning of the center of rotation of the mechanism with the anatomical center of rotation. We investigated the influence of misalignment between the anatomical and mechanism center of rotation (Fig. 5(a)) by shifting the center of rotation $\pm 2 \mathrm{~cm}$ in $\mathrm{x}$ - and $\mathrm{y}$-direction.

Also, the misalignment between the theoretical and implemented elastic band attachment points is investigated by varying $a_{l} \pm 20 \%$ with respect to $a_{2}$ (Fig. 5(b)), and varying $k_{1}$ $\pm 20 \%$ with respect to $k_{2}$ (Fig. 5(c)). Since $r_{1}$ and $r_{2}$ are equal as both distal attachment points are always moved simultaneously along the linear slider, we will not investigate the influence of ratio $r_{1}$ and $r_{2}$ on the balance.

2) Enclosed Angles: Equation (1) is compromised if either angle $\alpha$ and/or $\beta$ is changed such that the addition of these

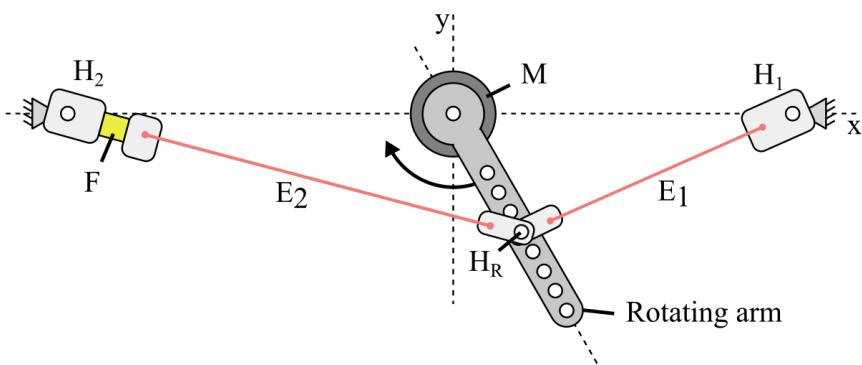

Fig. 6 Schematic top view of the experimental test rig to perform lifetime and stiffness measurements of elastic bands. A rotating arm is connected to the motor $(\mathrm{M})$ to continuously elongate and shorten two elastic bands $\left(\mathrm{E}_{1}\right.$ and $\left.\mathrm{E}_{2}\right)$ between limits $1_{\min }$ and $1_{\max }$. These limits can set by moving $\mathrm{H}_{1}$ and $\mathrm{H}_{2}$ along the $\mathrm{x}$-axis and by moving the $\mathrm{H}_{\mathrm{R}}$ along the rotating arm. A force sensor $(\mathrm{F})$ is connected to measure the force-length characteristic at predefined intervals.
TABLE IV SUBJECT CHARACTERISTICS

\begin{tabular}{|c|c|c|}
\hline & Subject 1 & Subject 2 \\
\hline Age & 54 & 19 \\
\hline Sex & M & M \\
\hline Condition & $\begin{array}{l}\text { Dysplasia and secundary } \\
\text { osteoarthritis with } \\
\text { involvement of capsule } \\
\text { and rotator cuff } \\
\text { musculature }\end{array}$ & $\begin{array}{l}\text { Hypermobility of } \\
\text { rotator cuff caused } \\
\text { by Ehlers-Danlos } \\
\text { syndrome }\end{array}$ \\
\hline Condition phase & Chronic & Chronic \\
\hline Affected shoulder & $\mathrm{R}$ & $\mathrm{R}$ \\
\hline Dominant side & $\mathrm{R}$ & $\mathrm{L}$ \\
\hline Length (cm) & 180 & 186 \\
\hline Weight (kg) & 92 & 72 \\
\hline
\end{tabular}

angles is not equal to $\pi$. In Fig. 5(d) the angle $\alpha$ is changed between $\pm 20 \%$. In Fig. 5(e) the vertical location of the distal attachment points on the upper arm is changed between $\pm 20 \%$. This influences angle $\beta$.

3) Zero-free Length: If the force-length relationship of the elastic bands changes over time, for example due to degradation of the elastomeric chains, the elastic bands may not have a zerofree length anymore. This will distort the balance of the system since (3) is violated. We measure the force-length characteristics with the experimental test setup described in the next paragraph.

A linear regression model is fitted to the force-length data according to:

$$
F=\beta_{0}+k x
$$

Here, $\beta_{0}$ is the intercept, $\mathrm{k}$ the slope of the linear fit and $F$ and $x$ are respectively the measured force and elastic band length.

We calculated the $95 \%$ confidence intervals of the intercept $\beta_{0}$ of the linear fit. The elastic bands show zero-free-length behavior if the value 0 is included in the confidence intervals of the intercept. The influence on the resulting balance can be determined by calculating the moment around the GHJ due to each band as a function of the flexion angle.

\section{Elastic bands characterization}

Two factors that influence the performance of the brace are the stiffness and lifetime of the elastic bands. An experimental test rig (Fig. 6) is developed to measure the force-length characteristics of the elastic bands over time during cyclic loading. From the data the stiffness can be computed. The system can also automatically detect failure of the elastic bands by analysis of sudden changes in the motor current. These data are used to compute the average lifetime. The setup includes LSB200 force sensors (Futek, USA) in series with elastic bands to measure the force during cyclic movements applied by an EC90 brushless rotary stage (Maxon Motor AG, Switzerland) connected to a rotating arm. The worst-case scenario involves cyclic stretching of the elastic bands between $150 \%$ and $350 \%$ of their length with a frequency of $1 / 3 \mathrm{~Hz}$. These lengths equals a shoulder flexion angle between $0^{\circ}$ and $90^{\circ}$ for a 50th percentile male, see also Table III. Four elastic bands are tested 
simultaneously of which two are connected to force sensors. Every 5 minutes force sensor data is collected during one complete cycle. Elastic bands of type 1 and 2 (see Table II) are tested. The test setup was placed in an air-conditioned room $\left(18^{\circ} \mathrm{C}\right)$ to ensure equal test conditions.

1) Stiffness: From the force-length relation of the elastic bands we can determine the stiffness in the zero-free-length range. A decreased stiffness will reduce the maximum force that the bands can apply to the arm. The stiffness of the elastic bands is derived from the slope $(k)$ of the linear regression model (4).

2) Lifetime: If the elastic bands fail during use, the user will experience an undesired, sudden change in the magnitude of the force applied to the arm. By evaluating the lifetime during a worst-case scenario we can determine the average time required to replace the elastic bands.

\section{Pilot Evaluation Methods}

Two patients with GHS are included in a pilot evaluation study to assess the functionality and subjective experiences of the shoulder subluxation support. Ethical approval for this study was obtained from the local Medical Ethics Committee.

A physiatrist of the Roessingh, Center for Rehabilitation (Enschede, NL) selected the participants for this evaluation. The characteristics of the participants are listed in Table IV. Both subjects are clinically diagnosed with GHS of approximately $1 \mathrm{~cm}$ upon palpation, and suffer from GHSrelated shoulder pain in rest and during movement. None of the participants had been prescribed a shoulder orthosis or other intervention prior to the study as none of the available commercially available orthoses were considered suitable for their condition and residual capabilities by their rehabilitation physician. Written informed consent was obtained from all participants before the study.

The pilot evaluation consisted of two sessions. During the first session ( $\sim 30 \mathrm{~min})$ several measurements of the patient's arm, shoulder and trunk were taken to customize the shoulder brace.

The aim of the second session ( $\sim 1$ hour) was to gain insight in the functionality and comfort of the brace through quantitative measures, and qualitative observations. First the forces in the elastic bands were assessed. The subjects are instructed to tension the elastic bands to a level that feels

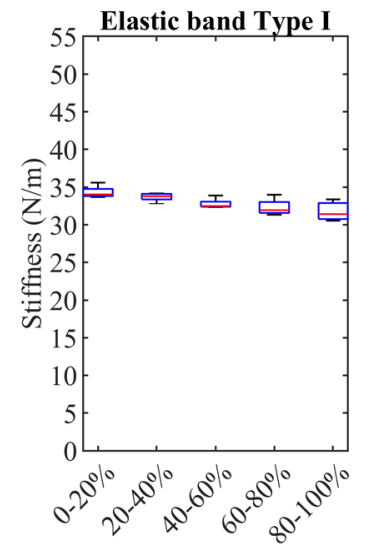

Lifetime (\%)

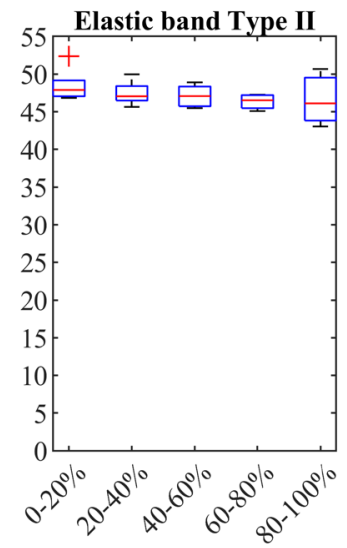

Lifetime (\%)
Fig. 7 Boxplots showing the stiffness $(\mathrm{N} / \mathrm{m})$ of the measured elastic bands type I (left) and type II (right) over bins of $\Delta 20 \%$ of their lifetime. comfortable, after which the lengths of the elastic bands were measured. The lengths are related to the applied forces according to (3). Then, the participants were asked to rate their average shoulder pain level during a normal day, and the minimum and maximum pain in the last 24 hours before the session on a scale from 0 to 10 . Also, qualitative remarks about aspects such as restriction during arm movements due to the brace, stability of the GHJ and comfort of the design were obtained during a structured interview. Additionally the donning and doffing time were recorded. Finally, the System Usability Scale (SUS) was conducted to subjectively assess usability. The provided SUS indices of the new design are important indicators during the development process. Subjects are asked to score the agreement or disagreement with 10 statements on a 5-point Likert scale. The results are translated to a score between 0 and 100 [28]. Higher scores represent a better usability.

\section{RESULtS}

\section{A. Attachment Point Locations}

Valid elastic band locations are identified that satisfy the conditions for static balance (1) to (3) and don't violate the proposed constraints (Table III).

For 90 percent of the adult population, the attachment points $\mathrm{P}_{1}$ and $\mathrm{P}_{2}$ are located on the red dashed line of Fig. 4. For the 50th percentile male, the solution is indicated with red dots in Fig. 4. This solution allows the users to freely move their arm between angles $\theta_{\mathrm{f}}=90^{\circ}$ and $\theta_{\mathrm{e}}=20^{\circ}$ without touching the shoulder bracket. Also, the spring attachment points stay close to the body within the specified limits.

\section{B. Conditions for Balance}

A detailed overview of the resulting moments due to shifts of model parameters can be found in Appendix I. A deviation from the optimal configuration of the elastic band attachment points of the shoulder brace will affect the equations for balance (1) to (3). For each model parameter change (see also Fig. 5), the effects on the balance of the system were investigated. In summary, the study showed that in neutral position, the alignment of the brace is most sensitive for shifts between the mechanism $\mathrm{CoR}$ and anatomical $\mathrm{CoR}$ in $\mathrm{x}$-direction. For example, a $2 \mathrm{~cm}$ shift leads to an additional moment of $0.5 \mathrm{Nm}$, (Fig 8A). Furthermore, a shift in y-direction of the CoR does not lead to an unbalance in neutral position (Fig.8B). However, the moment increases for larger flexion angles. Also, a vertical displacement of the distal attachment points (Fig. 8F), which occurs during tensioning of the elastic bands, does not create large additional moments $(<0.1 \mathrm{Nm})$.

\section{Elastic Bands Characterization}

1) Lifetime: For each elastic band tested, the number of cycles until failure is recorded. Elastic bands of type 1 have an average number of cycles until failure of $29 \times 10^{3} \pm 8.9 \times 10^{3}(\mathrm{n}=20)$. Type 2 bands have an average number of cycles until failure of $9.5 \times 10^{3} \pm 2.9 \times 10^{3}(\mathrm{n}=20)$. All elastic bands failed at the interface between pin and band.

The average number of arm movements of healthy individuals reported in literature (above $40^{\circ}$ with a duration between 1 and 5 s) is approximately between 100 and 120 
TABLE V

RESULTS OF THE PILOT EVALUATION

\begin{tabular}{|c|c|c|}
\hline & Subject 1 & Subject 2 \\
\hline Force elastic bands (\% of arm weight) & 71 & 83 \\
\hline Type of elastic band used & Type II & Type II \\
\hline $\begin{array}{l}\text { Restriction due to construction of brace } \\
(0-10)\end{array}$ & 0 & 1 \\
\hline $\begin{array}{l}\text { Restriction due to added resistance by } \\
\text { elastic bands }(0-10)\end{array}$ & 0 & 0 \\
\hline Pain score, min-max during last $24 \mathrm{~h}(0-10)$ & $0-6$ & $2-6$ \\
\hline Pain score, average of normal day $(0-10)$ & 4 & 5 \\
\hline Pain score without brace $(0-10)$ & 4 & 4 \\
\hline Pain score with brace $(0-10)$ & 1 & 4 \\
\hline SUS score $(0-100)$ & 97.5 & 92.5 \\
\hline
\end{tabular}

movements per hour [15],[19]. When the number of cycles until failure are compared to the average number of arm movements, we can conclude that the elastic band of type $I$ is able to withstand at least 30 days of usage in worst-case scenario (continuous stretching between $0^{\circ}$ and $90^{\circ}$ for 8 hours per day). For the type 2 elastic bands the average lifetime equals approximately 10 days of use in the extreme case.

2) Stiffness: The stiffness of the elastic bands of type I and type II is determined using a linear regression fit. The average stiffness of the elastic bands is plotted as a function of the lifetime binned over intervals of $20 \%$ in Fig. 7.

The average stiffness of the type I elastic bands drops from $34.3 \mathrm{~N} / \mathrm{m}$ to $31.7 \mathrm{~N} / \mathrm{m}$. This equals a decrease of $7.8 \%$. The average stiffness of the type II elastic bands drops from 48.5 $\mathrm{N} / \mathrm{m}$ to $46.6 \mathrm{~N} / \mathrm{m}$. This equals a decrease of $3.9 \%$. As a result, the maximum force that the elastic bands can generate will also decrease. The user can compensate for this decreased force by adding more elastic bands or stretch the bands to generate the same amount of supporting force.

\section{Pilot evaluation}

The results of the pilot evaluation are presented in Table V. 1) Applied force: The tension in the elastic bands was measured and scaled to the arm weight of the subjects. The arm weight was estimated from the body mass [27]. Subject 1 applied a force of $32 \mathrm{~N}$. This equals a percentage of $71 \%$ of his approximated arm weight. The tension in the elastic bands of subject 2 was $29 \mathrm{~N}$. This equals $83 \%$ of his approximated arm weight.

2) Glenohumeral stability: The glenohumeral stability was subjectively assessed. Both patients reported a feeling of increased stability of the shoulder when wearing the orthosis. Subject 2 mentioned that he could lift objects more easily due to this increased stability.

3) Restriction during movement: The restriction of the arm due to the construction of the brace during movements was scored on a scale from 0 to 10 by the subjects, where 0 indicates no restriction and 10 is a high level of restriction. Subject 1 reported no hindrance $(0 / 10)$ during voluntary arm movements. Subject 2 experienced very little hindrance (1/10) during voluntary arm movements.

Also, both subjects were asked to rate the additional effort they had to provide during movement due to resistance by the brace caused by possible misalignment on a scale from 0 (no added resistance) to 10 (high level of resistance). Both subjects reported no added resistance $(0 / 10)$ by the elastic bands during maximal arm flexion and abduction movements.

4) Pain: The least and worst shoulder pain experienced by subject 1 in the last 24 hours was respectively 0 and 6 . He reported an average pain during a normal day of 4 . Subject 2 experienced a pain between 2 and 6 in the last 24 hours. During a normal day his average pain score is 5 .

The instantaneous effect of the brace on the shoulder pain was assessed by scoring the pain without and with the shoulder brace. At the start of the second session, subject 1 experienced a pain of 4 . With the brace his pain score reduced to 1 . For subject 2 there was no difference in pain score without and with the orthosis. In both cases his pain score was 4 .

5) Comfort: Regarding comfort of the device, the subjects reported high pressures on the shoulder arc. The arm cuff stayed in place when applying tension to the elastic band. No irritation of the skin occurred. Subject 1 noted that it was difficult to attach the posterior elastic band to the shoulder bracket. Subject 2 mentioned that the comfort during seating could be improved since the harness of the brace is running over the spine and touches the back rest of a chair. Currently, the orthosis has a weight of $650 \mathrm{gr}$.

6) Usability: The average donning time of both participants over three trials is $86 \mathrm{~s}$. The average doffing time measured across three trials is $22 \mathrm{~s}$. Both participants completed the SUS for the shoulder brace after the second session. The SUS score of subject 1 was 97.5 and the score of subject 2 was 92.5 .

\section{DISCUSSION}

Patients with GHS and GHS-related shoulder pain may benefit from a shoulder brace that provides a restoring force to realign the GHJ. Current solutions are limited in that they impede the retaining range of motion of the upper arm. For these patients, a new shoulder subluxation support is developed that does not have the disadvantages of the currently available braces. The orthosis is applicable to a wide target population, including, but not limited to stroke, rotator cuff injury and neuromuscular diseases. Also, the orthosis can be used in a clinical and home setting to facilitate training and activities of daily living.

The brace contains two statically balanced zero-free length springs that realign the shoulder joint while providing a restoring glenoid-directed force. To guarantee the static balance of the system, and thus allow unrestricted arm movement within the retaining range of motion of the user, the shoulder brace should be aligned such that the equations for static balance (Eq. 1 to 3 ) hold. Misalignment of the brace is defined as the offset of one or more elastic band attachment point locations from the desired position. Providing clear instructions for the initial fitting by the orthotist is important, because only inaccurate brace fitting can result in a misalignment of the elastic bands, leading to a supporting force that is not directed toward the center of rotation of the shoulder joint. When misalignment occurs, the user will experience either a resistive or assistive force when the arm is kept in a certain position. The orthotist should in this case realign the elastic bands to match the conditions for static balance.

For each error source contributing to the misalignment of the brace, the effect on the balance of the system is investigated. 
Simulation results revealed that shifts in $\mathrm{x}$ - or $\mathrm{y}$-direction of the shoulder brace CoR with respect to the anatomical CoR have the most detrimental effects on the balance. However, even in these cases, the added moment due to misalignment is low $(<$ $0.5 \mathrm{Nm}$ ).

In our simulation, we determined the influence of one parameter at the time on the unbalance of the system. In practice, misalignment of the brace can violate multiple parameters of the conditions for static balance simultaneously. Depending on the specific configuration of the brace, the change of multiple parameters can either reinforce or reduce the unbalance.

Also, we only considered arm movements in the sagittal plane (flexion/extension). A detailed analysis of the balance in the frontal plane during abduction is presented in Appendix II. In the frontal plane the system acts as a gravity equilibrator, compensating the (partial) weight of the arm in abduction. Changing the location of the distal spring attachment points will change the magnitude of the abduction force provided by the mechanism.

The average lifetime of the elastic bands is determined during a worst-case test. The disposable elastic bands are very cheap and easily replaceable. Tests have confirmed that both types of elastic bands survive during a sufficient time before replacement of the bands is required. All bands failed at the attachment point. Improving this interface by reducing the friction between the attachment point and elastic band will probably extend the lifetime of the elastic bands.

In a pilot study with two participants several aspects of the shoulder orthosis were investigated. Both patients reported that the shoulder subluxation support provided a stabilizing force to the shoulder and, to a lesser extent, provided an immediate shoulder pain relief. The participants also addressed several issues regarding the comfort of the brace. Both subjects reported large reaction forces on the shoulder arc. The shoulder subluxation support reduces the stress by applying a force between the humerus and scapula. The magnitude of these reaction forces is directly related to the tension in the elastic bands. The comfort will likely be increased if the pressure distribution of the shoulder bracket is improved, for example, by using softer materials. Another improvement could be the reduction of weight. The results of the SUS suggest that the usability of the proposed system is promising. The device is likely to be accepted in daily activities for scores above 70 . Truly superior products score better than 90 [29]. A comparison with other orthoses is not possible, as the SUS scores of these devices are not available.

The conclusions presented in this study are based on quantitative and qualitative remarks about glenohumeral stability, pain, comfort and usability during a pilot evaluation with only two patients. Still, the results provide valuable insights and suggestions for improvements. A clinical study will be performed to further investigate the functionality and usability of the system. The shoulder pain will be evaluated after (medium) prolonged orthosis usage to assess the added benefit of the device for pain reduction. In this future study, we will also investigate the GHJ alignment using an imaging modality such as ultrasound, in order to objectively establish the re-positioning of the humeral head in the glenoid by the brace. This method has an advantage over conventional radiographs since there is no radiation involved.

\section{CONCLUSION}

In this study, a new shoulder subluxation support is presented for patients with GHS and GHS-related shoulder pain. The brace uses two statically balanced zero-free-length springs that provide an external force to the arm in the direction of the glenoid in different arm postures. Compared to existing shoulder braces, our design has the potential to reduce GHSrelated shoulder pain without impeding the range of motion of the user, even when the arm is moved away from the neutral position. Preliminary tests with two participants showed promising results.

\section{APPENDIX I}

A deviation from the optimal configuration of the elastic band attachment points of the shoulder brace will affect the equations for balance which are given by (1) to (3). For each model parameter change, the effects on the balance of the system are investigated.

The results of this sensitivity analysis are depicted in Fig. 8. In green the optimal configuration of the elastic band attachment points is shown. In this case the resulting moment is $0 \mathrm{Nm}$. The resulting moments can be visually compared to a reference moment Fig. 8(g). This is the moment required to lift the arm against gravity for each flexion angle. These results are discussed in the paragraphs below.

\section{A. Potential Energy}

A shift in the center of rotation with respect to the anatomical center of rotation in both $\mathrm{x}$ - and $\mathrm{y}$-direction was simulated. A shift in $\mathrm{x}$-direction (Fig. 8(a)) disturbs the balance when the arm is in neutral position and decreases to approximately $0 \mathrm{Nm}$ at a flexion angle of $90^{\circ}$. For the y-direction (Fig. 8(b)) the opposite occurs. A slight shift does not affect the resulting moment in neutral position. However, the moment added by the brace increases when the arm is flexed. For a shift of $2 \mathrm{~cm}$ a resulting moment of approximately $0.45 \mathrm{Nm}$ occurs at a flexion angle of $90^{\circ}$.

The effect of changing length $a_{1}$ (with respect to $a_{2}$ ) on the resulting moment can be seen in Fig. 8(c). From this graph we can see that a maximum moment of approximately $0.2 \mathrm{Nm}$ occurs around $30^{\circ}$ shoulder flexion for an increase in length of $a_{1}$ of $20 \%$.

The stiffness of the anterior and posterior elastic bands are varied. In Fig. 8(d) the results are shown. A maximum moment of approximately $0.2 \mathrm{Nm}$ is observed at $30^{\circ}$ flexion.

\section{B. Enclosed Angles}

Changing angle $\alpha$ will affect (1), which is required for balance of the system. The results are shown in Fig. 8(e). It can be seen that in neutral position the arm will experience a moment due to the misalignment of the brace. When the arm is flexed, the moment reduces to $0 \mathrm{Nm}$ at about $30^{\circ}$ after which it increases again to a maximum of about $0.3 \mathrm{Nm}$ at $90^{\circ}$ flexion.

Changing the vertical location (L) of attachment points $\mathrm{D}_{1}$ and $\mathrm{D}_{2}$ on the upper arm will alter angle $\beta$. The resulting moment (Fig. 8(f)) shows a similar trend as was seen when 
varying angle $\alpha$. The maximum moment occurs at $90^{\circ}$ flexion and is approximately $0.25 \mathrm{Nm}$.

\section{Zero-free-length}

The force-length characteristics of the elastic bands type I and type II are shown in Fig. 9 averaged over their lifetime.
For each data set, a linear regression fit was computed. A zero-free-length is achieved when the $95 \%$ confidence interval of the intercept includes the value 0. The dashed lines in Fig. 9 indicate the average length range in which the elastic bands show zero-free-length behavior.

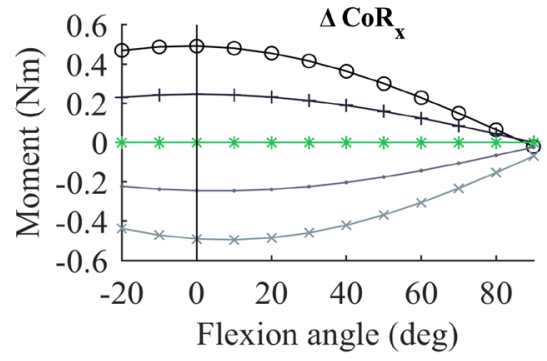

(a)

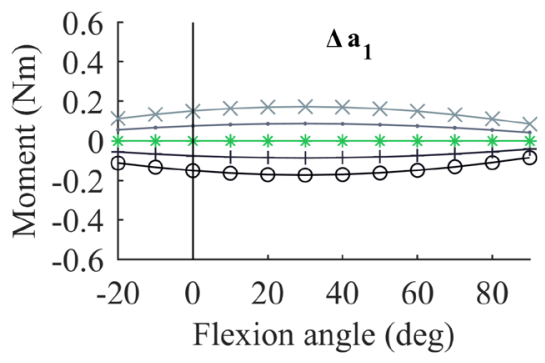

(c)

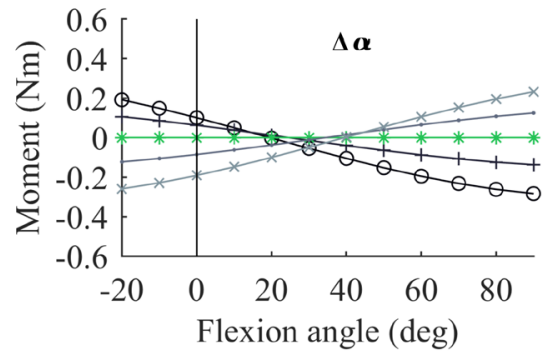

(e)
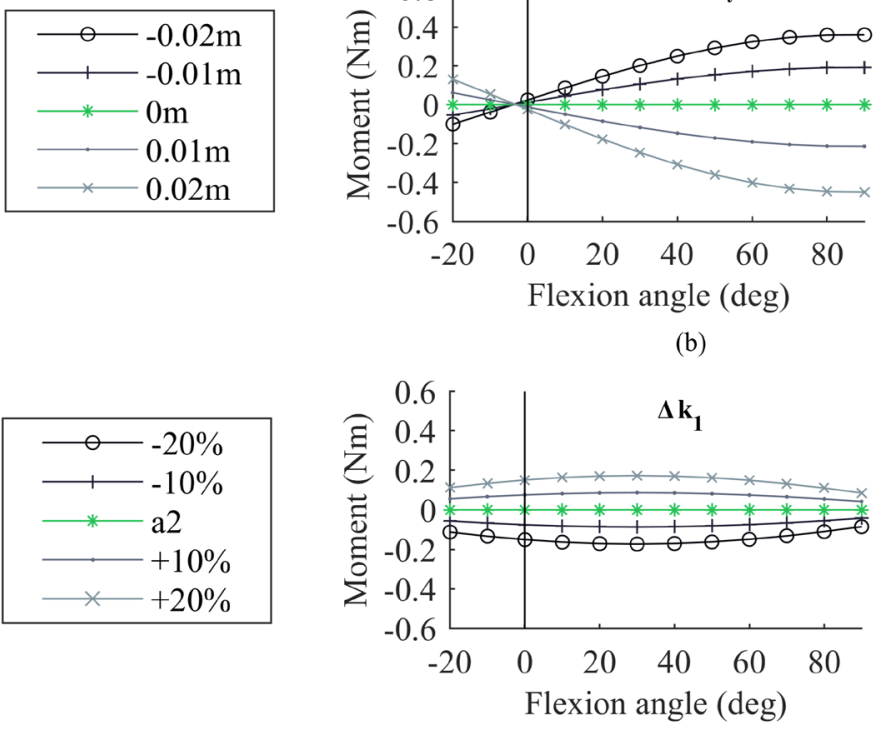

(d)

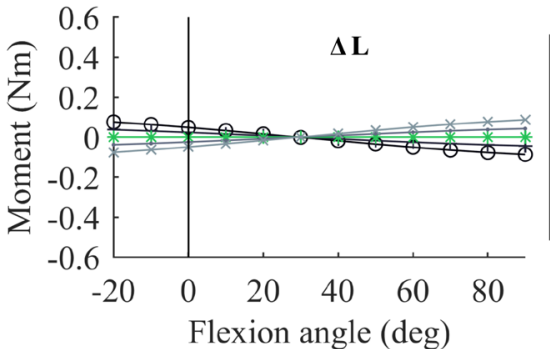

(f)

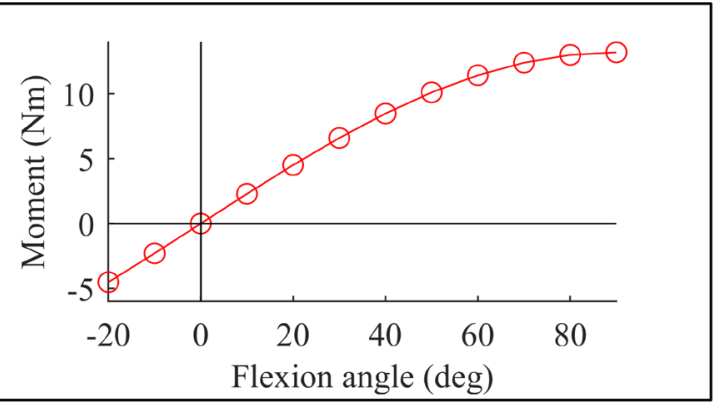

(g)

Fig. 8 Moments caused by the shoulder brace for a 50th percentile male when parameters of the shoulder brace are varied with respect to the optimal configuration. The parameters are graphically depicted in Fig. 4. In the optimal configuration the system is balanced. In this configuration, the moment caused by the shoulder brace is $0 \mathrm{Nm}$ over the complete movement from $-20^{\circ}$ to $90^{\circ}$ flexion (green lines). A negative value represents a moment that counteracts arm flexion. a) Xcoordinate of CoR changed with $\pm 2 \mathrm{~cm}$. b) Y-coordinate of CoR changed with $\pm 2 \mathrm{~cm}$. c) Distance $a_{1}$ changed $\pm 20 \%$ with respect to $a_{2}$. d) Stiffness $k_{1}$ changed \pm $20 \%$ with respect to $\mathrm{k}_{2}$. e) Angle $\alpha$ changed $\pm 20 \%$ with respect to optimal configuration $\left(145^{\circ}\right)$. f) Length $\mathrm{L}$ changed $\pm 20 \%$ with respect to optimal configuration $(0.2 \mathrm{~m})$. g) Moment required by a 50th percentile male to lift his arm against gravity for different flexion angles. (Note: the scale of the y-axis has changed.) 


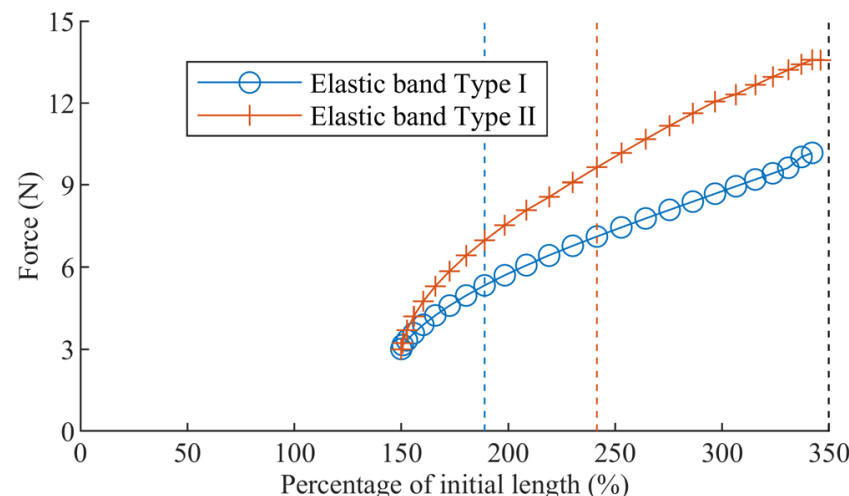

Fig. 9 Force-length characteristics of elastic bands of type I and II averaged over their lifetime. The type I elastic band shows zero-free-length behavior between the blue and black dashed line. The type II elastic band shows zerofree-length behavior between the red and black dashed line.

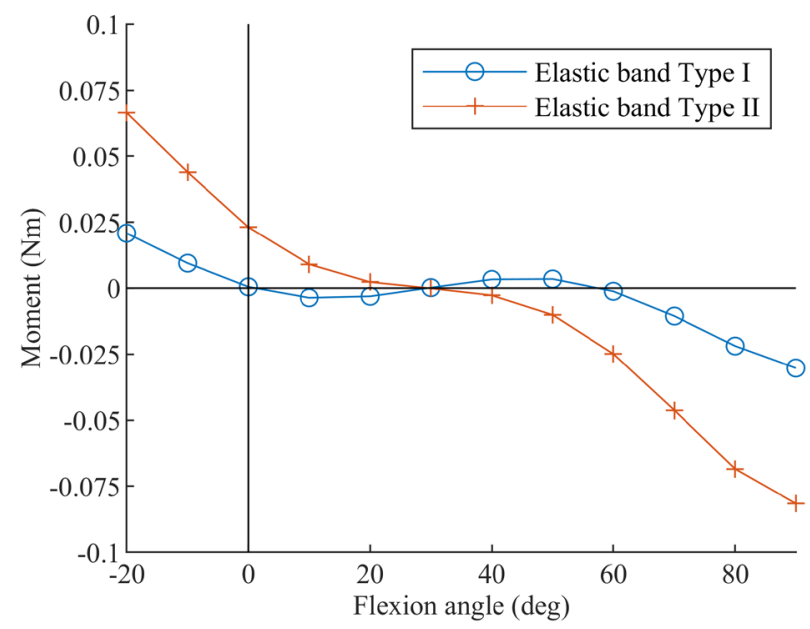

Fig. 10 Moments caused by the non-zero-free-length characteristics of the elastic bands for a 50th percentile male.

For the type I elastic bands the zero-free-length behavior is observed between $180 \%$ and $350 \%$ of their initial length. The type II elastic bands show zero-free-length behavior between $230 \%$ and $350 \%$. In the shoulder support, the elastic bands are used between $150 \%$ and $350 \%$, which is outside their linear range. This will affect the balance of the system. The moment that is added by the elastic bands is plotted in Fig. 10 as a function of the flexion angle. For the type I elastic bands the maximum moment is approximately $0.03 \mathrm{Nm}$. For the type II elastic bands the maximum moment is approximately $0.08 \mathrm{Nm}$. Compared to the moment required to move the arm through the range of motion (see also, Fig. 8g), this additional moment is negligible.

\section{APPENDIX II}

In the manuscript, only the balance of the arm in the sagittal plane has been considered (Fig. 11(a)). The moments created by the anterior and posterior elastic bands are balanced, such that no net moment is introduced around the CoR of the shoulder joint. The locations of the attachment point $\mathrm{A}$ and $\mathrm{P}$ are shown in the frontal plane, see Fig. 11(b).

In the frontal plane, the combination of the two elastic bands acts as a gravity equilibrator. Depending on the distance $\left(a_{A}\right.$ and

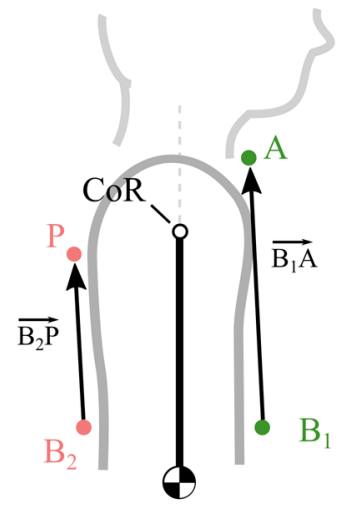

sagittal plane

(a) frontal plane, $45^{\circ}$ abd.

(b)

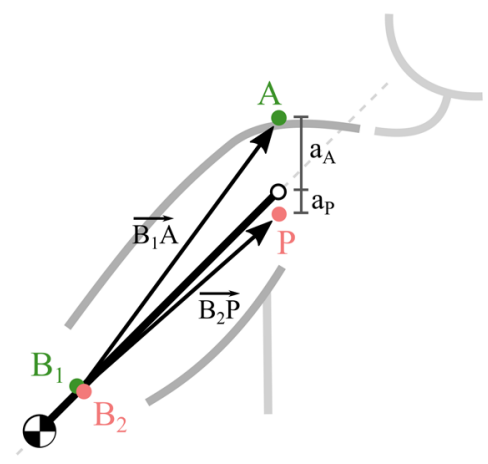

Fig. 11 (a) Sagittal view of the arm showing the CoR, the elastic band anterior (A) and posterior (P) attachment points (red and green dots) and force vector (red and green arrow). (b) Frontal view with the arm in $45^{\circ}$ abduction. In abduction, a net abducting moment is introduced as a result of both force vectors $F_{A}$ and $F_{P}$.

$a_{P}$, Fig. 11(b)) of points A and $\mathrm{P}$ with respect to the CoR, each of the bands contribute to exactly compensating (part of) the weight of the arm. An elastic band that is attached above the CoR (e.g. point A in Fig. 11) will contribute to an abducting moment of the arm, whereas an elastic band that is attached below the CoR (e.g. point P in Fig. 11) will contribute to an adducting moment of the arm.

The condition for static balance of this gravity equilibrator in the frontal plane is [24]:

$$
m g L=a k r
$$

Here $m g$ is the force caused by the arm weight, acting on distance $L$ from the CoR. The elastic bands with stiffness $k$ are attached at a distance $r$ from the CoR to the upper arm and a distance $a$ to the trunk. See also Fig. 12 for a schematic overview. From the equation it can be seen that the amount of weight that is compensated $(\mathrm{m})$ is, among others, determined by the position of the attachment points $\mathrm{A}$ and $\mathrm{P}$.

Given the optimized position of attachment points $\mathrm{A}$ and $\mathrm{P}$ in the sagittal plane, the combination of elastic bands will compensate approximately $6 \%(220 \mathrm{~g})$ of the arm weight of a

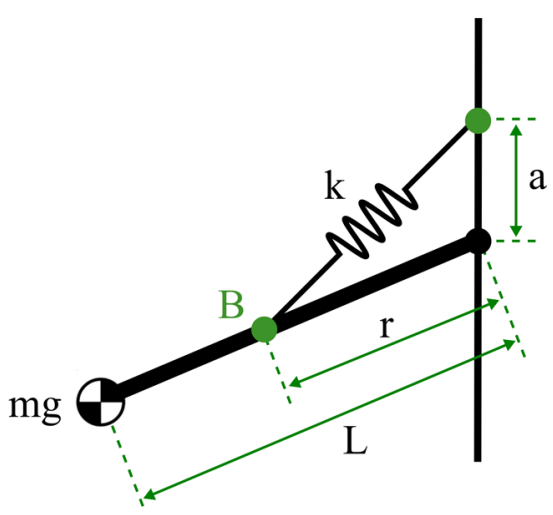

Fig. 12. Schematic overview showing the basic components of the gravity equilibrator. Force $m g$ acting on a distance $L$ from the shoulder joint is balanced by a zero-free-length spring with stiffness $k$ attached at distance $a$ and $\mathrm{r}$ from the rotational center. 


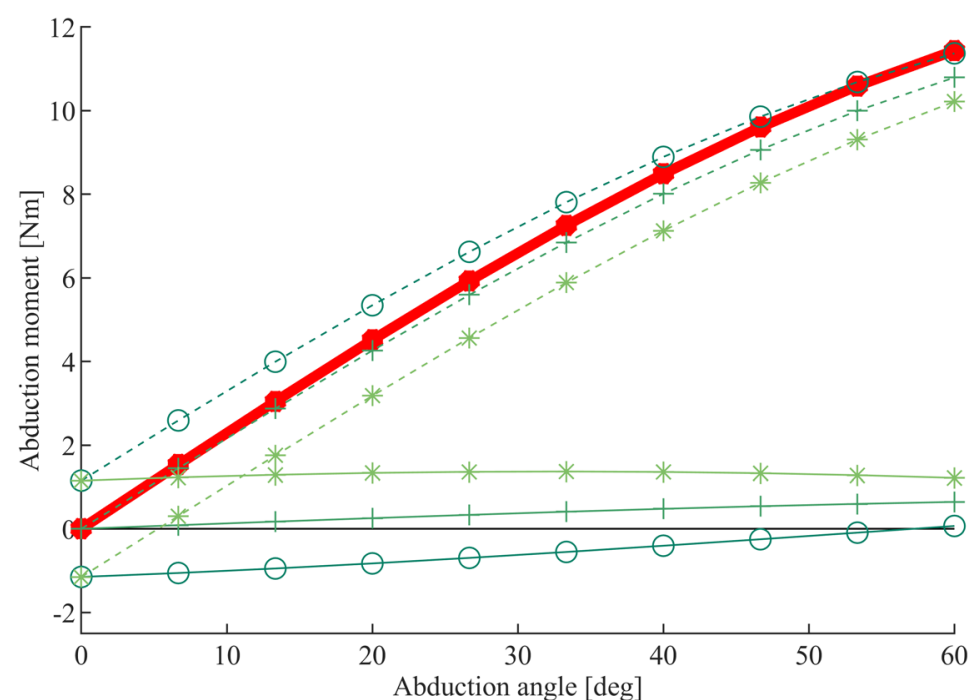

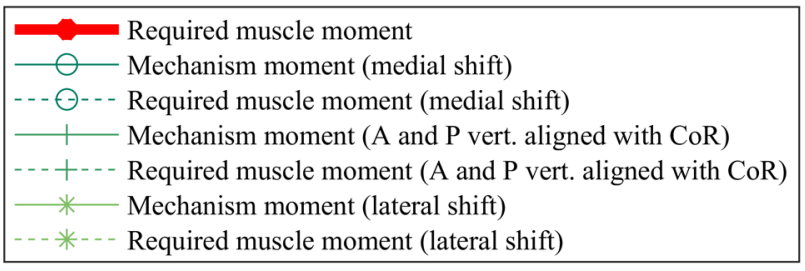

Fig. 13. Abduction moment versus angle plots for three locations of the anterior (A) and posterior (P) elastic band attachment points. If points A and $P$ are vertically aligned with the CoR in the frontal plane, the abduction moment caused by the elastic bands increases for larger abduction angles of the arm (+). Moving the attachment points in lateral $(*)$ or medial (o) direction causes an offset of the abduction moment (solid lines). This moment now counteracts (in case of a lateral shift) or assists (in case of a medial shift) the patient's voluntary abduction moment, as can be seen by comparing the moment required to lift the arm (solid red line) and the resulting moments when the elastic bands act on the arm (dashed lines).

$50^{\text {th }}$ percentile adult male throughout the entire range of motion in the frontal plane (abduction angle).

In general, having an assistive abducting moment can be beneficial, as patients with reduced muscle strength are aided (to a small extent) in abduction. Also, patients with continuous tension on the rotator cuff musculature due to glenohumeral subluxation may find assistance by the brace during abduction beneficial. If desired by the patient, the abducting moment can be increased by moving attachment point locations $\mathrm{A}$ and/or $\mathrm{P}$ up. However, this will deteriorate the balance of the arm in the sagittal plane during flexion. The abducting moment can also be increased by moving attachment points $\mathrm{A}$ and $\mathrm{P}$ laterally in the frontal plane. However, then the system is no longer a perfect gravity equilibrator, which means that the relative amount of weight compensation changes with the abduction angle. This is shown in Fig. 13. If A and P are shifted medially ('o', solid line), additional effort is required to overcome the adducting moment caused by the bands. But if $\mathrm{A}$ and $\mathrm{P}$ are shifted laterally ('*', solid line), the assisting moment helps the patient to abduct his arm.

\section{ACKNOWLEDGMENT}

The authors would like to thank Freek Tonis (Hankamp Rehab), Reinout van Vliet (Roessingh, Center for Rehabilitation) and JohnJohn de Koning (Roessingh Rehabilitation Technique) for providing valuable insights during the design process. The authors would also like to thank Koen Heuver and Marijn Peters for their assistance during the development of the prototype and evaluation of the brace.

\section{REFERENCES}

[1] K. Moore, A. Dalley, "Shoulder anatomy," in Clinically Oriented Anatomy, 4th ed., Philadelphia: Lippincott Williams and Wilkins, 1999, pp. 788-795.

[2] D. Jenkins, "Shoulder and upper limb," in Functional Anatomy of the Limbs and Back, 9th ed., Maryland Heights, MO: Saunders Elsevier, 2009 , pp. 86-89.
[3] T. Ikai, K. Tei, K. Yoshida, S. Miyano, K. Yonemoto, (1998). Evaluation and treatment of shoulder subluxation in hemiplegia: relationship between subluxation and pain. American Journal of Physical Medicine \& Rehabilitation. 77(5), pp. 421-426.

[4] L. Turner-Stokes, D. Jackson (2002, May). Shoulder pain after stroke: a review of the evidence base to inform the development of an integrated care pathway. Clinical rehabilitation. 16(3), pp. 276-298.

[5] L. T. Andersen (1985). Shoulder Pain in Hemiplegia. American Journal of Occupational Therapy. 39(1), pp. 11-19.

[6] M. Nadler, M. M. H. H. Pauls (2016). Shoulder orthoses for the prevention and reduction of hemiplegic shoulder pain and subluxation: Systematic review. Clinical Rehabilitation. 31(4), pp. 444-453.

[7] D. Stolzenberg, G. Siu, E. Cruz (2012). Current and Future Interventions for Glenohumeral Subluxation in Hemiplegia Secondary to Stroke. Topics in Stroke Rehabilitation. 19(5), pp. 444-456.

[8] L. Ada, A. Foongchomcheay, C. G. Canning (2005). Supportive devices for preventing and treating subluxation of the shoulder after stroke. Stroke. 36(8), pp. 1818-1819.

[9] K. Li, N. Murai, S. Chi (2013). Clinical reasoning in the use of slings for patients with shoulder subluxation after stroke: a glimpse of the practice phenomenon in California. OTJR: Occupation, Participation and Health. 33(4), pp.228-235.

[10] S. Hesse, C. Herrmann, A. Bardeleben, M. Holzgraefe, C. Werner, I. Wingendorf, et al. (2013). A new orthosis for subluxed, flaccid shoulder after stroke facilitates gait symmetry: A preliminary study. Journal of Rehabilitation Medicine. 45(7), pp. 623-629.

[11] M. Hartwig, G. G. Gelbrich, B. Griewing (2012). Functional orthosis in shoulder joint subluxation after ischaemic brain stroke to avoid posthemiplegic shoulder-hand syndrome: a randomized clinical trial. Clinical rehabilitation. 26(9), pp. 807-816.

[12] K. Dieruf, J. L. Poole, C. Gregory, E. J. Rodriguez, C. Spizman (2005). Comparative effectiveness of the GivMohr sling in subjects with flaccid upper limbs on subluxation through radiologic analysis. Archives of Physical Medicine and Rehabilitation. 86(12), pp. 2324-2329.

[13] A. van Bladel, G. Lambrecht, K. M. Oostra, G. Vanderstraeten, D. A. Cambier (2017). A randomized controlled trial on the immediate and long-term effects of arm slings on shoulder subluxation in stroke patients. European Journal of Physical and Rehabilitation Medicine. 53(3), pp. 400-409.

[14] A. Morley, A. Clarke, S. English, S. Helliwell (2002). Management of the Subluxed Low Tone Shoulder. Physiotherapy. 88(4), pp. 208-216.

[15] B. Coley, B. M. Jolles, A. Farron, K. Aminian (2008). Arm position during daily activity. Gait and Posture. 28(4), pp. 581-587.

[16] K. Meijer, J. Annegarn, V. L. Passos, H. H. Savelberg, A. M. Schols, E. F. Wouters, et al. (2014). Characteristics of daily arm activities in patients with COPD. European Respiratory Journal. 43(6), pp. 1631-1641. 
[17] G. D. G. Langohr, J. P. Haverstock, J. A. Johnson, G. S. Athwal (2018). Comparing daily shoulder motion and frequency after anatomic and reverse shoulder arthroplasty. Journal of Shoulder and Elbow Surgery. 27(2), pp. 325-332.

[18] R. Chapman, M. Torchia, J. E. Bell, D. Van Citters (2019). Assessing Shoulder Biomechanics of Healthy Elderly Individuals During Activities of Daily Living Using Inertial Measurement Units: High Maximum Elevation Is Achievable but Rarely Used. Journal of Biomechanical Engineering. 141(4), pp. 1-7.

[19] M. Acuna, T. Amasay, A. R. Karduna (2010). The reliability of side to side measurements of upper extremity activity levels in healthy subjects. BMC Musculoskeletal Disorders. 11(1), pp. 168.

[20] S. H. Collins, P. G. Adamczyk, A. D. Kuo (2009). Dynamic arm swinging in human walking. Proceedings of the Royal Society B: Biological Sciences. 276(1673), pp. 3679-3688.

[21] J. D. Ortega, L. A. Fehlman, C. T. Farley (2008). Effects of aging and arm swing on the metabolic cost of stability in human walking. Journal of Biomechanics. 41(16), pp. 3303-3308.

[22] M. Paci, L. Nannetti, L. A. Rinaldi (2005). Glenohumeral subluxation in hemiplegia: An overview. The Journal of Rehabilitation Research and Development. 42(4), pp. 557.

[23] P. Mckee, A. Rivard, "Foundations of Orthotic Intervention," in Rehabilitation of the Hand and Upper Extremity, 6th ed. Philadelphia, PA: Elsevier; 2011, pp. 1565-1580.

[24] J. L. Herder, "Energy-free systems: theory, conception, and design of statically balanced spring mechanisms," Ph.D. dissertation, Delft University of Technology, Delft, 2001.

[25] A. G. Dunning, "Slender spring systems," Ph.D. dissertation, Delft University of Technology, Delft, 2016.

[26] C. D. Fryar, Q. Gu, C. L. Ogden (2012, Oct.). Anthropometric reference data for children and adults: United States, 2007-2010. Vital Health and Statistics. 11(252).

[27] P. de Leva (1996, Sept.). Adjustments to Zatsiorsky-Seluyanov's segment inertia parameters. Journal of Biomechanics. 29(9), pp. 1223-1230.

[28] J. Brooke, "SUS: A 'Quick and Dirty' Usability Scale," in Usability evaluation in Industry. London: Taylor \& Francis; 1996, pp. 189-194.

[29] A. Bangor, P. T. Kortum, J. T. Miller (2008). An empirical evaluation of the system usability scale. International Journal of Human-Computer Interaction. 24(6), pp. 574-594. 